\title{
W boson polarization in vector boson scattering at the LHC
}

\author{
Ezio Maina*, Alessandro Ballestrero and Giovanni Pelliccioli \\ Department of Physics - University of Torino and INFN Sez. Torino \\ E-mail: mainadto.infn.it, ballestrerodto.infn.it, \\ gpellicceto.infn.it
}

Measuring the scattering of longitudinally-polarized vector bosons represents a fundamental test of the mechanism of Electroweak Symmetry Breaking.

In addition to the challenges provided by low rates and large backgrounds, there are conceptual issues which need to be clarified for the definition of a suitable signal. Since vector bosons are unstable and can only be observed through their decay products, the polarization states interfere among themselves. Moreover, already at tree level, there are diagrams which cannot be interpreted as production times decay of EW bosons but are necessary for gauge invariance.

We discuss a possible way to define a cross section for polarized $W$ 's, dropping all non resonant diagram, and projecting on shell the resonant ones, thus, preserving gauge invariance. In most cases, the sum of polarized distributions reproduces accurately the exact results. In the absence of cuts, the polarization fractions obtained with this procedure agree with the results of a standard projection on Legendre polynomials. While the latter cannot be employed in the presence of selection cuts on the charged leptons, a comparison of the data with singly polarized templates allows the extraction of the polarization fractions in a realistic environment.

For this work, the possibility of generating Vector Boson Scattering events with singly polarized $W$ 's has been introduced into Phantom.

The European Physical Society Conference on High Energy Physics

5-12 July, 2017

Venice

${ }^{*}$ Speaker. 


\section{Introduction}

The discovery of a scalar particle [1,2], whose properties are compatible with those of the Higgs Boson [3, 4], is a confirmation of the Standard Model description of ElectroWeak Symmetry Breaking (EWSB). In the SM, vector bosons acquire mass through their couplings to the Higgs field. The Higgs is also essential in Vector Boson Scattering (VBS), to tame the growth, at high energy, in the longitudinally-polarized sector.

The experimental results on VBS in Run 1 at the LHC suffer from low statistics. In the near future, Run 2 will collect many more data. Still, the expected effects are very small, therefore, improvements in theoretical predictions and experimental strategies will be necessary.

Understanding theoretically how to separate the polarizations of the W's in VBS is required in order to separate them in the data. The basic tool has been known for a long time: each of the three on-shell polarizations results in a specific decay distribution of the charged leptons. Two issues, however, remain unresolved:

- Since the W's are unstable particles, the decays of the indidual polarizations interfere among themselves. These interference contributions cancel exactly only when an integration over the full azimuth of the lepton is performed. Acceptance cuts, however, inhibit collecting data over the full angular range and interference effects are non zero. In addition, cuts affect differently the three polarizations and, therefore, the full decay distribution is not simply the sum of the distributions corresponding to single polarizations.

- With few exceptions, ElectroWeak boson production processses are described by amplitudes including non resonant diagrams, which cannot be interpreted as production times decay of any vector boson. These diagrams are essential for gauge invariance and cannot be ignored. For them, separating polarizations is simply unfeasible.

In this talk we discuss how to disentangle the polarizations of the $W$ in the simple case of $W^{+} W^{-}$production in VBS when both vector boson decay leptonically.

\section{W boson polarization and angular distribution of its decay products}

Let's consider an hadronic Standard Model tree level process, at $\mathscr{O}\left(\alpha_{E M}^{2} \alpha_{S}^{n}\right)$, involving an external $W^{+}$which decays leptonically. The amplitude can be written, in the Unitary Gauge, as

$$
\mathscr{M}=\mathscr{M}_{\mu} \frac{i}{k^{2}-M^{2}+i \Gamma_{w} M}\left(-g^{\mu v}+\frac{k^{\mu} k^{v}}{M^{2}}\right)\left(\frac{-i g}{2 \sqrt{2}} \bar{\psi}_{l} \gamma_{v}\left(1-\gamma^{5}\right) \psi_{v_{l}}\right) .
$$

The polarization tensor can be expressed in terms of four polarization vectors:

$$
-g^{\mu v}+\frac{k^{\mu} k^{v}}{M^{2}}=\sum_{\lambda=1}^{4} \varepsilon_{\lambda}^{\mu}(k) \varepsilon_{\lambda}^{v^{*}}(k) .
$$

In a frame in which the off-shell $W$-boson propagates along the $z$-axis, with momentum $\kappa$, energy $E$ and invariant mass $\sqrt{Q^{2}}=\sqrt{E^{2}-\kappa^{2}}$, the polarizations read: 


$$
\begin{aligned}
& \varepsilon_{L}^{\mu}=\frac{1}{\sqrt{2}}(0,-1,-i, 0)(\text { left }), \quad \varepsilon_{R}^{\mu}=\frac{1}{\sqrt{2}}(0,+1,-i, 0)(\text { right }) \\
& \varepsilon_{0}^{\mu}=(\kappa, 0,0, E) / \sqrt{Q^{2}} \text { (longitudinal), } \quad \varepsilon_{A}^{\mu}=\sqrt{\frac{Q^{2}-M^{2}}{Q^{2} M^{2}}}(E, 0,0, \kappa) \text { (auxiliary) }
\end{aligned}
$$

The decay amplitudes of the $W$ depend on its polarization. In the rest frame of the $\ell v$ pair, they are:

$$
\mathscr{M}^{\mathscr{D}}{ }_{0}=i g \sqrt{2} E \sin \theta, \quad \mathscr{M}^{\mathscr{D}}{ }_{R / L}=i g E(1 \pm \cos \theta) e^{ \pm i \phi},
$$

where $(\theta, \phi)$ are the charged lepton angles relative to the boson direction. The decay amplitude for the auxiliary polarization is zero. Hence, each physical polarization is uniquely associated with a specific angular distribution of the charged lepton.

Defining a polarized production amplitude $\mathscr{M}_{\lambda}^{\mathscr{P}}=\mathscr{M}_{\mu} \varepsilon_{\lambda}^{\mu}$, the full amplitude can be written as:

$$
\mathscr{M}=\sum_{\lambda=1}^{3} \mathscr{M}^{\mathscr{P}} \lambda \frac{i}{k^{2}-M^{2}+i \Gamma_{w} M} \mathscr{M}^{\mathscr{D}} \lambda=\sum_{\lambda=1}^{3} \mathscr{M}_{\lambda}^{\mathscr{F}} \lambda
$$

where $\mathscr{M}_{\lambda}^{\mathscr{F}}$ is the full amplitude with a single polarization for the intermediate $W$.

The squared amplitude becomes:

$$
\underbrace{|\mathscr{M}|^{2}}_{\text {coherent sum }}=\underbrace{\sum_{\lambda}\left|\mathscr{M}^{\mathscr{F}} \lambda\right|^{2}}_{\text {incoherent sum }}+\underbrace{\sum_{\lambda \neq \lambda^{\prime}} \mathscr{M}^{\mathscr{F} *} \mathscr{M}^{\mathscr{F}} \lambda^{\prime}}_{\text {interference terms }} .
$$

The interference terms in eq.(2.7) are not, in general, zero. They cancel only when the squared amplitude is integrated over the full range of the angle $\phi$, or, equivalently, when the charged lepton can be observed for any value of $\phi$. Since this is never possible in practice, interference among polarization states needs to be taken into account.

If we denote by $d \sigma(\theta, X) / d \cos \theta$ the integral over $\phi$ of the fully differential cross section, where $\theta, \phi$ are the $W$ decay variables in the boson rest frame and $X$ stands for all additional phase space variables, one can write, using eq.(2.5),

$$
\frac{1}{\sigma(X)} \frac{d \sigma(\theta, X)}{d \cos \theta}=\frac{3}{8}(1 \mp \cos \theta)^{2} f_{L}+\frac{3}{8}(1 \pm \cos \theta)^{2} f_{R}+\frac{3}{4} \sin ^{2} \theta f_{0}
$$

where the upper sign is for $W^{+}$and the lower sign for $W^{-}$, and $f_{L}, f_{0}$ and $f_{R}$ represent the left, longitudinal and right polarization fractions, respectively.

If eq.(2.8) holds, the polarized components can be extracted from the angular distribution by a projection on the first three Legendre polinomials.

In order to separate the polarized components in the data, it is necessary to compute the individual amplitudes $\mathscr{M}^{\mathscr{F}}{ }_{\lambda}$ in eq.(2.6), which requires making the substitution $\sum_{\lambda^{\prime}} \varepsilon_{\lambda^{\prime}}^{\mu} \varepsilon_{\lambda^{\prime}}^{v^{*}} \rightarrow \varepsilon_{\lambda}^{\mu} \varepsilon_{\lambda}^{\nu *}$. in the $W$ propagator. This possibility has been introduced in PHANTOM [5]. 


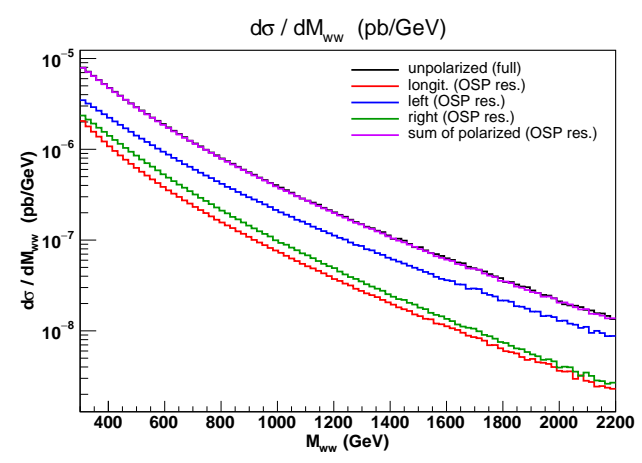

(a) $W W$ invariant mass

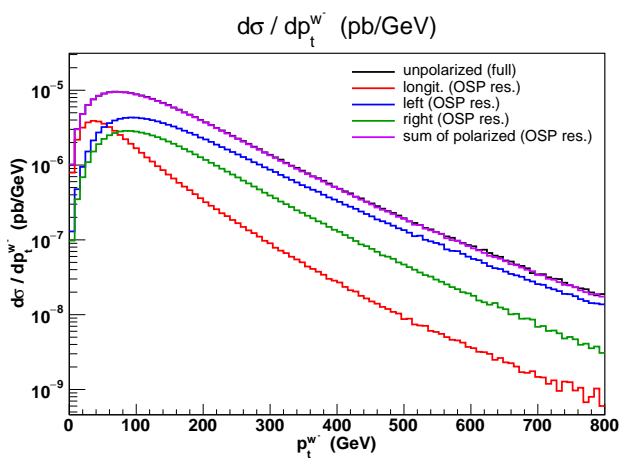

(b) $p_{t}^{W^{-}}$

Figure 1: Differential cross-sections: comparison between unpolarized generations based on the exact amplitude and the incoherent sum of the polarized generations with OSP which take into account only the resonant diagrams.

\section{Separating the resonant contribution: On Shell Projection}

We now turn to reactions which contain non resonant contributions, in particular to those in which two lepton pairs, $e^{-} \bar{v}_{e}$ and $\mu^{+} v_{\mu}$, are produced. There are diagrams which contain only one, space like, $W$ propagator. The only way to proceed, in order to define amplitudes with definite $W$ polarization, is to devise an approximation to the full result that only involves double resonant diagrams.

We have chosen an On shell Projection (OSP) method, similar to the procedure employed for the calculation of EW radiative corrections to $W^{+} W^{-}$production in refs.[6]. This can be realized as a completely gauge invariant approximation.

It consists in substituting the momentum of the $\ell v$ pair in the calculation of $\mathscr{M}^{\mathscr{P}} \lambda$ and $\mathscr{M}^{\mathscr{D}} \lambda$ in eq.(2.6) with a momentum on the $W$ mass shell, while the denominator in the $W$ propagator is left untouched. However, this projection is not uniquely defined. In order to have an unambiguous prescription we have chosen to conserve: the total four-momentum of the $W W$ system (thus, also $M_{W W}$ is conserved); the direction of the two $W$ bosons in the $W W$ center of mass frame; the angles of each charged lepton, in the corresponding $W$ center of mass frame, relative to the boson direction in the lab.

\section{Validating the approximation in the absence of cuts on the charged leptons}

In this section we compare the differential distributions of the $W W$ invariant mass, fig. 1(a) and the transverse momentum of the $e^{-} v_{e}$ pair, fig. 1(b) obtained from the exact matrix element and those obtained from the incoherent sum of three OSP distributions. The negatively charged intermediate $W$ boson is polarized while the positively charged one remains unpolarized. Only doubly resonant diagrams are included. No lepton cut is applied. Our aim is to show that for most distributions the sum of polarized distributions reproduces well the exact result and to explore the differences between the individual polarized distributions as a tool to separate them in the data.

In fig. 1 the black curves refers to the exact result and the violet ones to the sum of polarized distributions. The individual distributions are shown in red (longitudinal), blue (left) and green 


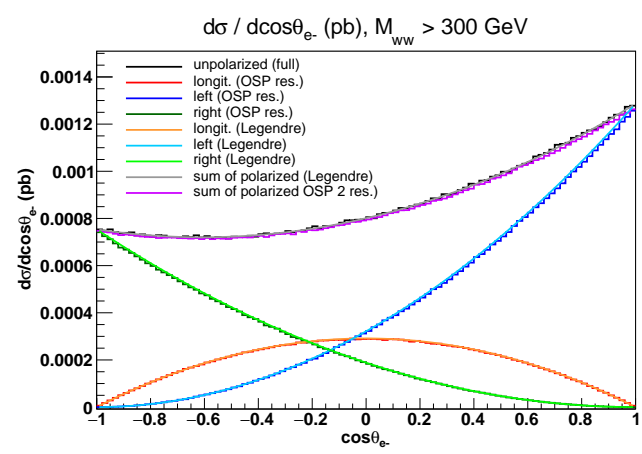

(a) $\cos \theta_{e}$

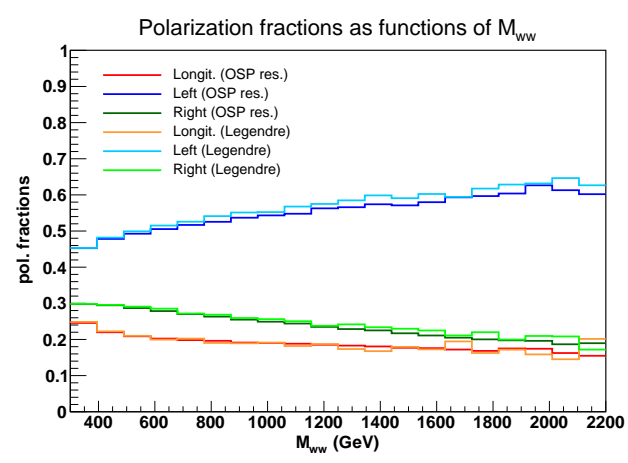

(b) Polarization fractions

Figure 2: Distribution of $\cos \theta$ in the $W$ reference frame (left), polarization fractions as functions of $M_{w w}$ (right).

(right). The exact cross section is $1.748 \mathrm{fb}$ while the incoherent sum of singly polarized cross sections is $1.731 \mathrm{fb}$, which differs from the full result by about $1 \%$. The incoherent sum of three OSP distributions agrees very well with the full result.

Fig. 1(b) shows that longitudinally polarized $W$ 's have a markedly softer $p_{t}$ spectrum in comparison with transversely polarized ones. In fact they dominate for $p_{t}^{W}<50 \mathrm{GeV}$.

In fig. 2, on the left, we show the distribution of the decay angle of the electron in the reference frame of the $e^{-} v_{e}$ pair integrated over the full range $M_{w w}>300 \mathrm{GeV}$. On the right we present the polarization fractions as a function of the invariant mass of the four leptons, which provides additional information since the distributions in fig. 2(a) are dominated by events with relatively small $M_{w w}$.

The colors are as in fig. 1: black refers to the exact result, violet to the incoherent sum of polarized distributions, red to the longitudinal polarization, blue and green to the left and right polarization, respectively.

The results of the expansion on Legendre polynomials are shown as lighter shade smooth lines in fig. 2(a) and show that the distributions from the polarized generations, in the absence of cuts on the charged leptons, have the expected functional form and that the polarization fractions extracted from the exact results are in excellent agreement with the polarized distributions.

Fig. 2(b) presents the polarization fractions of the $W^{-}$as a function of $M_{W W}$. The darker lines show the ratio of the individual polarized cross sections to the exact result in each $M_{W W}$ bin; the lighter lines are obtained from a bin by bin expansion on Legendre polynomials of the exact result. The two methods agree. Fig. 2(b) shows that, in the SM, $W^{-}$'s produced in VBS are mainly left-handed. The fraction of left-polarized $W^{-}$'s increases with increasing $M_{W W}$. The fraction of longitudinal and right-handed $W$ 's are roughly the same at large invariant masses. The longitudinal fraction is almost constant at about $20 \%$. The right-handed component decreases slightly from approximatly $30 \%$ at $M_{W W}=400 \mathrm{GeV}$ to just above $20 \%$ at $M_{W W}=2000 \mathrm{GeV}$.

\section{Leptonic cuts and their effects}

In fig. 3 and fig. 4 we show how the distributions presented above are modified by the introduc- 
tion of standard acceptance cuts on both charged leptons. We require $p_{t}^{\ell}>20 \mathrm{GeV}$ and $\left|\eta^{\ell}\right|<2.5$.

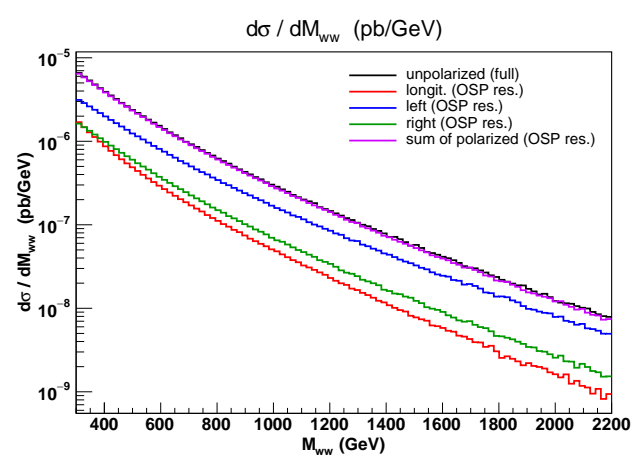

(a) $W W$ invariant mass

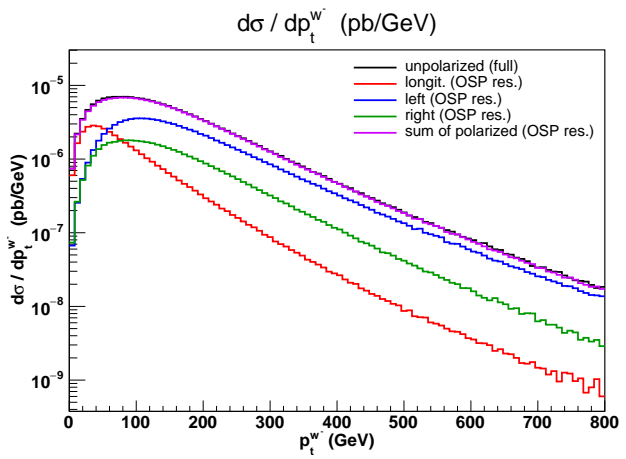

(b) $p_{t}^{W^{-}}$

Figure 3: Differential cross-sections: comparison between unpolarized generation and sum of the polarized generation. $p_{t}^{\ell}>20 \mathrm{GeV},\left|\eta^{\ell}\right|<2.5$.

The exact cross section is 1.411(1) fb, the coherent sum of OSP polarized amplitudes gives 1.401(1) fb, while the incoherent sum of singly polarized cross sections is $1.382(1) \mathrm{fb}$, which differs from the full result by about $2 \%$. A number of features are worth noticing. The cross section from the coherent sum of polarized amplitudes is very close to the incoherent sum of cross sections, which indicates that the interference among polarizations is generally small. The exact cross section is well reproduced by both approximations.

The incoherent sum of three OSP distributions agrees well with the full result for the $W W$ invariant mass, fig. 3(a) and the transverse momentum of the $e^{-} v_{e}$ pair, fig. 3(b). The individual polarizations are not affected equally by the cuts. Typically, the cross section for right-handed $W$ 's is reduced the most, followed by the cross section for longitudinally polarized $W$ 's. Left-handed $W$ bosons seem to be the least sensitive to acceptance cuts.

The full angular distribution, fig. 4(a), is approximated within a few percent, over the full range, by the sum of the unpolarized results. The exact result, shown by the black histogram, however, is not of the form of eq.(2.8). This becomes clear expanding the full result on the first

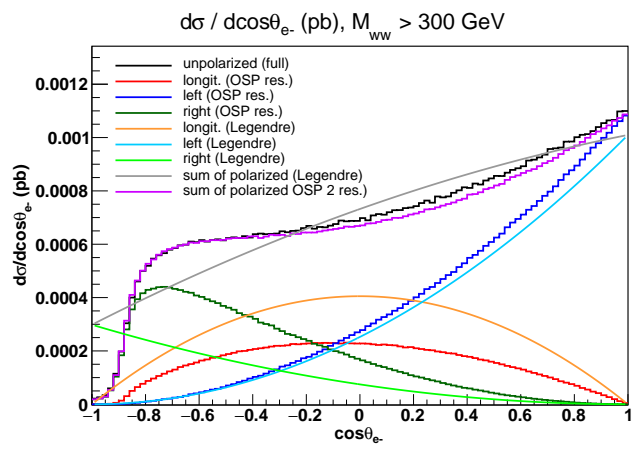

(a) $\cos \theta_{e}$

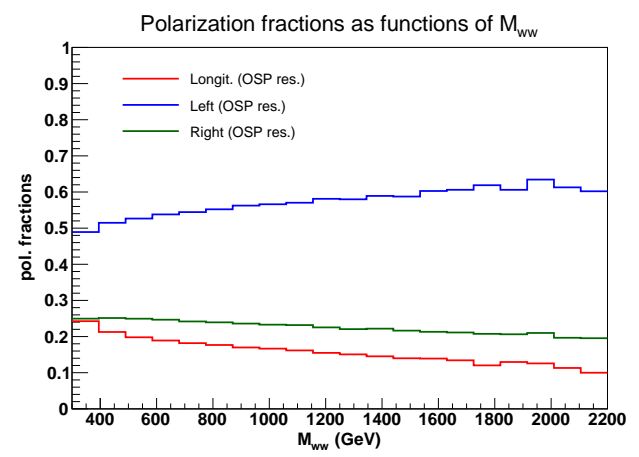

(b) Polarization fractions

Figure 4: Distribution of $\cos \theta$ in the $W$ reference frame (left), polarization fractions as functions of $M_{w w}$ (right). 
three Legendre polynomials, which yields the blue, green and orange smooth curves in fig. 4. Their sum is the smooth black curve which fails to describe the correct distribution. Even in the presence of cuts, the fraction of longitudinally polarized $W^{-}$'s is well above $10 \%$.

The fact that the exact distribution is well described by the incoherent sum of the polarized differential distributions allows for a measurement of the polarization fractions within a single model, even in the presence of cuts on the charged leptons, using Montecarlo templates for the fit.

\section{Conclusions}

In this talk we have shown a way to measure the polarization of a $W$ boson in VBS events. It is based on the observation that the set of doubly resonant diagrams, suitably projected on shell to preserve gauge invariance, approximate well the full result and allow an expansion in terms of amplitudes in which each final state $W$ has a definite polarization. This procedure agrees with the standard approach based on Legendre polynomials in the absence of cuts on the decay leptons. When acceptance cuts are imposed on the leptons, and the Legendre polynonmials procedure fails, it is possible to extract the polarization fractions using singly polarized SM Monte Carlo templates. Additional details can be found in ref. [7].

\section{References}

[1] G. Aad et al. [ATLAS Collaboration], Phys. Lett. B 716 (2012) 1 doi:10.1016/j.physletb.2012.08.020 [arXiv:1207.7214 [hep-ex]].

[2] S. Chatrchyan et al. [CMS Collaboration], Phys. Lett. B 716 (2012) 30 doi:10.1016/j.physletb.2012.08.021 [arXiv:1207.7235 [hep-ex]].

[3] G. Aad et al. [ATLAS and CMS Collaborations], Phys. Rev. Lett. 114 (2015) 191803 doi:10.1103/PhysRevLett.114.191803 [arXiv:1503.07589 [hep-ex]].

[4] G. Aad et al. [ATLAS and CMS Collaborations], JHEP 1608 (2016) 045 doi:10.1007/JHEP08(2016)045 [arXiv:1606.02266 [hep-ex]].

[5] A. Ballestrero, A. Belhouari, G. Bevilacqua, V. Kashkan and E. Maina, Comput. Phys. Commun. 180 (2009) 401 doi:10.1016/j.cpc.2008.10.005 [arXiv:0801.3359 [hep-ph]].

[6] A. Denner, S. Dittmaier, M. Roth and D. Wackeroth, Nucl. Phys. B 587 (2000) 67 doi:10.1016/S0550-3213(00)00511-3 [hep-ph/0006307].

[7] A. Ballestrero, E. Maina and G. Pelliccioli, arXiv:1710.09339 [hep-ph]. 